\title{
Gastrointestinal symptoms associated with severity of coronavirus disease 2019 (COVID-19): a pooled analysis
}

\author{
Brandon Michael Henry ${ }^{1} \cdot$ Maria Helena Santos de Oliveira ${ }^{2} \cdot$ Justin Benoit ${ }^{3} \cdot$ Giuseppe Lippi $^{4}$
}

Received: 19 March 2020 / Accepted: 26 March 2020 / Published online: 17 April 2020

(c) Società Italiana di Medicina Interna (SIMI) 2020

Keywords Nausea $\cdot$ Vomiting $\cdot$ Diarrhea $\cdot$ Coronavirus $\cdot$ COVID-19

Dear Editor,

The identification of clinical predictors of progression towards severe form is urgently needed to enable risk stratification and optimize resource allocation in coronavirus disease 2019 (COVID-19) pandemic. Recently, gastrointestinal shedding of viral RNA detected in fecal samples has been reported in both children and adults [1,2]. This may be unsurprising given the high expression of viral host receptor angiotensin converting enzyme 2 (ACE2) in gastrointestinal tract [3]. In a clinical study of 138 COVID-19 patients, Wang et al. reported that $10 \%$ experienced gastrointestinal symptoms 1-2 days prior the onset of fever and dyspnea [4]. Therefore, we aim here to explore whether or not patients presenting with abdominal pain, nausea, and vomiting, or diarrhea may be at increased odds of the severe form of COVID-19.

We performed an electronic search in Medline (PubMed interface) and the Chinese National China National Knowledge Infrastructure (CNKI) with the keywords "nausea" OR "vomiting" OR "diarrhea" in all fields AND "coronavirus 2019” OR “COVID-19” OR “2019-nCoV” OR “SARSCoV-2", between 2019 and present time (i.e., March 15,

Brandon Michael Henry

Brandon.henry@cchmc.org

1 Cardiac Intensive Care Unit, The Heart Institute, Cincinnati Children's Hospital Medical Center, 3333 Burnet Avenue, Cincinnati, OH 45229, USA

2 Department of Statistics, Federal University of Parana, Curitiba, Brazil

3 Department of Emergency Medicine, University of Cincinnati, Cincinnati, OH, USA

4 Section of Clinical Biochemistry, Department of Neuroscience, Biomedicine and Movement, University of Verona, Verona, Italy
2020), with no language restrictions. Title, abstract, and of all full text of all identified items were analyzed, and those providing the prevalence of gastrointestinal symptoms in adult ( $>18$ years of age) COVID-19 patients with clinically validated definition of "severe disease" (i.e., patients needing mechanical ventilation, vital life support, and intensive care unit admission) were included in a pooled analysis. No exclusion criteria were applied. The reference list of all retrieved documents was also hand-searched (through forward and backward citation tracking) for identifying additional eligible studies. Articles in Chinese were screened by a healthcare professional fluent in both Chinese and English. When data describing gastrointestinal symptoms were identified, the article was translated into English to enable data collection. As the data set was limited and included case series, no study risk of bias or publication bias assessment was performed.

A pooled analysis was finally performed, encompassing the calculation of odds ratio (OR) and $95 \%$ confidence interval of abdominal pain, nausea, and vomiting in COVID19 patients with or without severe disease. The statistical analysis was carried out using MetaXL, software Version 5.3 (EpiGear International Pty Ltd., Sunrise Beach, Australia) with an inverse variance model. The study was carried out in accordance with the declaration of Helsinki and with the term of local legislation. As data were publicly available, no ethics approval was required.

After duplicate screening, a total number of 49 documents were initially identified, 37 of which ought to be excluded, because they were review articles $(n=13)$, did not report data on COVID-19 disease $(n=14)$, did not provide the prevalence of gastrointestinal symptoms in COVID-19 patients with or without severe disease $(n=7)$, or used mortality to define patient groups $(n=3)$. Two additional studies could be identified from the reference list of selected articles. Thus, the pooled analysis finally included 10 studies, 
with a total sample of 1989 confirmed COVID-19 patients, 598 of which (30.1\%) were classified as having severe disease [4-13]. All studies reported data as symptoms at onset or admission, except for two studies in which timing of symptoms was unclear $[8,11]$.

The results of the pooled analysis are shown in Fig. 1. Abdominal pain, which was reported in three studies $(n=731)[4,5,11]$ was significantly associated with increased COVID-19 severity (OR 3.93 [95\% CI 1.64-9.38), $I^{2}=0 \%$, Cochran's $Q, p=0.45$ ). Nausea and vomiting were reported in seven studies $(n=1924)[4,5,8-11,13]$ and were found to be significantly associated with a marginally increased odds of severe COVID-19 (OR 1.65 [95\%
CI 1.06-2.57), $I^{2}=0 \%$, Cochran's $\left.Q, p=0.78\right)$. However, diarrhea, reported in nine studies $(n=1974)$ [4-9, 11-13], was not associated with disease severity (OR 1.24 [95\% CI $0.90-1.71), I^{2}=0 \%$, Cochran's $\left.Q, p=0.83\right)$. Heterogeneity was not observed in any analysis (all $I^{2}=0 \%$ ).

Based on this investigation of early COVID-19 data, abdominal pain was found to be associated with a near fourfold increased odds of severe COVID-19, whilst a marginally significant increased prevalence has also been observed for nausea and vomiting, but not for diarrhea. We hypothesize that abdominal pain may be related to the degree of viral replication in the gut, with increased severity of illness observed related to a high viral load and subsequent viremia.
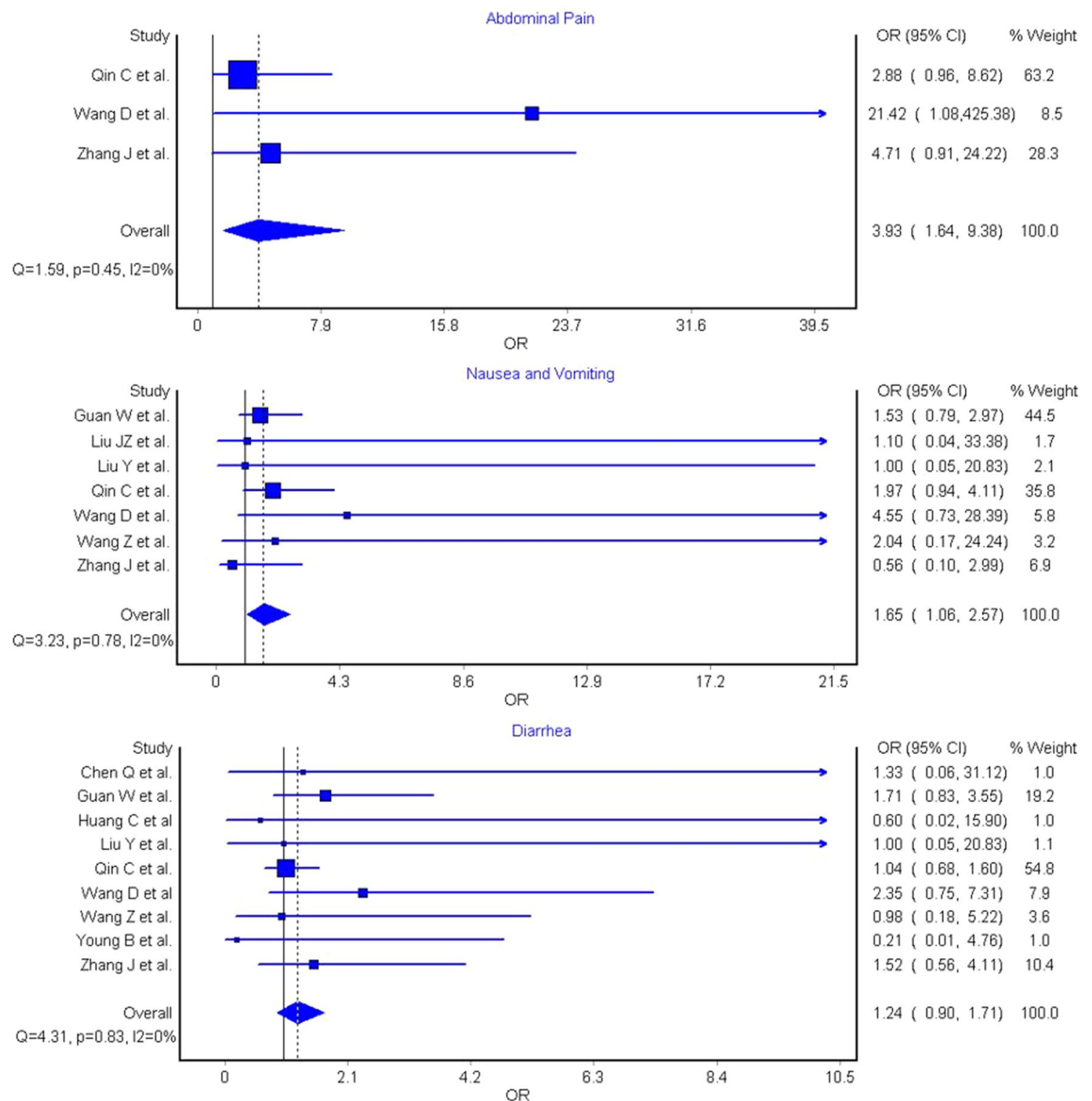

$\begin{array}{lc}\text { OR }(95 \% \mathrm{Cl}) & \% \text { Weight } \\ 1.33(0.06,31.12) & 1.0 \\ 1.71(0.83,3.55) & 19.2 \\ 0.60(0.02,15.90) & 1.0 \\ 1.00(0.05,20.83) & 1.1 \\ 1.04(0.68,1.60) & 54.8 \\ 2.35(0.75,7.31) & 7.9 \\ 0.98(0.18,5.22) & 3.6 \\ 0.21(0.01,4.76) & 1.0 \\ 1.52(0.56,4.11) & 10.4 \\ 1.24(0.90,1.71) & 100.0 \\ & \end{array}$

Fig. 1 Odds ratio (OR) and 95\% confidence interval (95\% CI) of abdominal pain, nausea/vomiting, and diarrhea between coronavirus disease 2019 (COVID-19) patients with or without severe disease 
We suggest that abdominal pain may be used as clinical predictor of more severe disease and be included in future risk stratification algorithms.

\section{Compliance with ethical standards}

Conflict of interst The authors declare no conflict of interests.

Statement of human and animal rights This study does not contain human participants or animals.

Informed consent For this type of study, informed consent in not required.

\section{References}

1. Zhang W, Du R-H, Li B et al (2020) Molecular and serological investigation of 2019-nCoV infected patients: implication of multiple shedding routes. Emerg Microbes Infect 9:386-389. https:// doi.org/10.1080/22221751.2020.1729071

2. Cai J, Xu J, Lin D et al (2020) A Case Series of children with 2019 novel coronavirus infection: clinical and epidemiological features. Clin Infect Dis. https://doi.org/10.1093/cid/ciaa198

3. Xu H, Zhong L, Deng J et al (2020) High expression of ACE2 receptor of 2019-nCoV on the epithelial cells of oral mucosa. Int J Oral Sci 12:1-5. https://doi.org/10.1038/s41368-020-0074-X

4. Wang D, Hu B, Hu C et al (2020) Clinical characteristics of 138 hospitalized patients with 2019 novel Coronavirus-infected pneumonia in Wuhan, China. JAMA. https://doi.org/10.1001/ jama.2020.1585
5. Zhang J-J, Dong X, Cao Y-Y et al (2020) Clinical characteristics of 140 patients infected with SARS-CoV-2 in Wuhan, China. Allergy. https://doi.org/10.1111/all.14238

6. Young BE, Ong SWX, Kalimuddin S et al (2020) Epidemiologic features and clinical course of patients infected with SARS-CoV-2 in Singapore. JAMA. https://doi.org/10.1001/jama.2020.3204

7. Huang C, Wang Y, Li X et al (2020) Clinical features of patients infected with 2019 novel coronavirus in Wuhan, China. Lancet 395:497-506. https://doi.org/10.1016/S0140-6736(20)30183-5

8. Guan W-J, Ni Z-Y, Hu Y et al (2020) Clinical characteristics of coronavirus disease 2019 in China. N Engl J Med. https://doi. org/10.1056/NEJMoa2002032

9. Liu Y, Yang Y, Zhang C et al (2020) Clinical and biochemical indexes from 2019-nCoV infected patients linked to viral loads and lung injury. Sci China Life Sci 63:364-374. https://doi. org/10.1007/s11427-020-1643-8

10. Liu J, Chen C, Xia S (2020) Clinical analysis of 15 cases of new coronavirus pneumonia. Henan J Prevent Med 3:161-164

11. Qin C, Zhou L, Hu Z et al (2020) Dysregulation of immune response in patients with COVID-19 in Wuhan, China. Clin Infect Dis. https://doi.org/10.1093/cid/ciaa248

12. Chen N, Zhou M, Dong X et al (2020) Epidemiological and clinical characteristics of 99 cases of 2019 novel coronavirus pneumonia in Wuhan, China: a descriptive study. Lancet 395:507-513. https://doi.org/10.1016/S0140-6736(20)30211-7

13. Wang Z, Yang B, Li Q et al (2020) Clinical features of 69 cases with coronavirus disease 2019 in Wuhan, China. Clin Infect Dis. https://doi.org/10.1093/cid/ciaa272

Publisher's Note Springer Nature remains neutral with regard to jurisdictional claims in published maps and institutional affiliations. 